\title{
Näherungslösungen für die Form kernmagnetischer Signale bei Impulsexperimenten in Festkörpern
}

\author{
G. SiEgLE \\ I. Physikalisches Institut der Universität Stuttgart \\ (Z. Naturforsch. 23 a, 1194-1201 [1968]; eingegangen am 18. April 1968)
}

\begin{abstract}
NMR transients in solids have been calculated using the method of Lowe and Norberg ${ }^{1}$ and its extension by Powles and Strange ${ }^{2}$. Some remarks are given concerning the evaluation of the formal solution for the signal after several pulses.

So far the influence of the pulse durations and of the longitudinal relaxation is neglected usually, higher terms of the power series expansion of the signals were discussed only with respect to corrections of their amplitudes. Some remarkable differences between these calculations and new experimental results to be reported here are explained - at least in principle - avoiding these limitations:

The maximum amplitude of the echo after two $90^{\circ}$-pulses (phase difference of their modulations $\Delta \varphi=90^{\circ}$ ) occurs not exactly at a time twice the pulse distance, the deviation is explained considering the duration of the pulses and the decay of the echo envelope.

After two pulses with $\Delta \varphi=0^{\circ}$ an echo was found which should disappear only in an ideal twospin system. For a three pulse sequence the influence of the longitudinal relaxation is described; and in a multi-pulse sequence the calculated modulation of the amplitude of successive echoes was checked by experiments.
\end{abstract}

In einer vorausgegangenen Arbeit ${ }^{3}$ wurden die möglichen Kernsignale in Festkörpern mit einer Kernspinsorte (Spin $I=1 / 2$ ) bei Impulsexperimenten angegeben. Die Ausgangsformel zu ihrer Berechnung ist nach dem Verfahren von Lowe und NorBERG $^{1}$ und dessen Erweiterung auf mehrere Impulse durch Powles und Strange ${ }^{2}$ aus der Bewegungsgleichung der Dichtematrix abgeleitet. Danach wird die meßbare Signalspannung $U$ nach $n$ Impulsen der Dauern $\tau_{a n}$, die zu den Zeiten $0, \tau_{1}{ }^{\prime}, \tau_{2}{ }^{\prime} \ldots$ eingestrahlt werden (Abb. 1), zur Meßzeit $t$ bei exakter Resonanzjustierung $\left(\omega_{0}=-\gamma_{\mathrm{I}} H_{0}\right)$

$$
\begin{aligned}
U\left(\begin{array}{c}
\alpha_{1} \ldots \alpha_{n} \\
\varphi_{1} \ldots \varphi_{n}
\end{array}\right)= & \left(C / \operatorname{Sp} I_{x}^{2}\right) \operatorname{Sp}\left(I_{x} \cos \omega_{0} t-I_{y} \sin \omega_{0} t\right) \exp \left\{-(i / \hbar) H^{I I} \tau_{n}\right\} \\
& \cdot J\left(\begin{array}{l}
\alpha_{n} \\
\phi_{n}
\end{array}\right) \ldots \exp \left\{-(i / \hbar) H^{I I} \tau_{1}\right\} J\left(\begin{array}{c}
\alpha_{1} \\
\phi_{1}
\end{array}\right) \\
& \cdot I_{z} J^{-1}\left(\begin{array}{c}
\alpha_{1} \\
\psi_{1}
\end{array}\right) \exp \left\{(i / \hbar) H^{I I} \tau_{1}\right\} \ldots J^{-1}\left(\begin{array}{c}
\alpha_{n} \\
\varphi_{n}
\end{array}\right) \exp \left\{(i / \hbar) H^{I I} \tau_{n}\right\}
\end{aligned}
$$

( $C=$ Konstante, $\gamma_{I}=$ gyromagnetisches Verhältnis der Spins $I, H_{0}=$ zeitlich konstantes äußeres Magnetfeld, $H^{I I}=$ dipolare Wechselwirkung der Spins, $J=\mathrm{Im}$ pulsoperator, $\alpha_{n}=$ Impulslänge, $\varphi_{n}=$ Phase der Impulsmodulation bez. des kohärenten Senders).

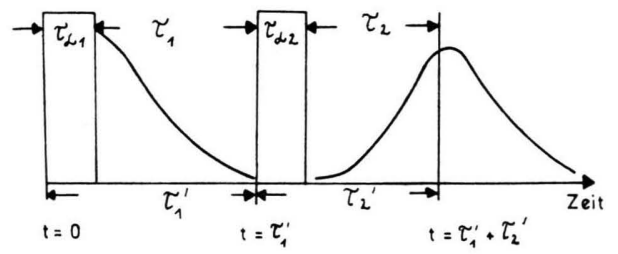

Abb. 1. Definition der im Text verwendeten Zeiten bei Doppelimpulsfolgen (für mehrere Impulse sinngemäße Erweiterung). $\tau_{1}^{\prime}=\tau_{\alpha 1}+\tau_{1}=$ „Impulsabstand“", $\tau_{2}{ }^{\prime}=\tau_{\alpha 2}+\tau_{2}=$ Abstand des Beobachtungszeitpunkts vom Beginn des zweiten Impulses.

1 I. J. Lowe u. R. E. Norberg, Phys. Rev. 107, 46 [1957].

2 J. G. Powles u. J. H. Strange, Proc. Phys. Soc. London 82 , 6 [1963].
Die wichtigsten Voraussetzungen zur Ableitung und Auswertung von Gl. (1) in ${ }^{3}$ sind

1. $H^{I l}$ sei zeitunabhängig (d. h. nur säkulare Anteile $H^{I I}=\sum_{i<j} a_{i j} I_{i} I_{j}+b_{i j} I_{i z} I_{j z}=A+B$, vgl. z. B. ${ }^{4}$ ); andere Wechselwirkungen werden nicht berücksichtigt.

2. Die Dauern der Impulse seien vernachlässigbar kurz, so daß $H^{I I}$ während der Impulse nicht berücksichtigt zu werden braucht.

3. Die zur Auswertung von Gl. (1) durchgeführte Reihenentwicklulng kann zur Bestimmung der prinzipiellen Signaleigenschaften bereits nach den in den Zeiten $\tau$ quadratischen Gliedern abgebrochen werden.

3 G. Siegle, Z. Naturforsch. 23 a, 91 [1968].

4 A. Abragam, The Principles of Nuclear Magnetism, Clarendon Press, Oxford 1961. 
In der vorliegenden Arbeit sollen einige Beispiele genannt werden, bei denen bemerkenswerte Abweichungen von den nach ${ }^{3}$ erwarteten Ergebnissen gefunden wurden. Es wird gezeigt, wie durch Abbau der obigen Voraussetzungen die Gültigkeit von Gl. (1) bzw. der daraus abgeleiteten Formeln so erweitert werden kann, daß eine Beschreibung des experimentell gefundenen Verhaltens der Kernmagnetisierung möglich wird. Da die Auswertung aber in jedem Fall als Reihenentwicklung durchgeführt wird, ist die Úbereinstimmung zwischen Rechnung und Experiment allerdings stets durch die geringe Zahl der berechneten Entwicklungsglieder beschränkt.

Nach einigen Bemerkungen zur Auswertung von Gl. (1) werden beschrieben:

die Form des Festkörperechos nach zwei $90^{\circ}$-Impulsen mit Phasenverschiebung $\Delta \varphi=90^{\circ} \mathrm{der}$ Impulsmodulationen; das Signal nach 2 gleichphasig modulierten Impulsen;

die Amplitude der Kernsignale einer Mehrimpulsfolge;

der Einfluß der Spin-Gitter-Relaxation.

Alle zugehörigen Experimente wurden an Protonen in einkristallinem Gips $\left(\mathrm{CaSO}_{4} \cdot 5 \mathrm{H}_{2} \mathrm{O}\right)$ und dem Paraffin $\mathrm{C}_{13} \mathrm{H}_{28}$ bei $28 \mathrm{MHz}$ durchgeführt.

\section{Zur Auswertung der Ausgangsgleichung (1)}

Gl. (1) wird in der Regel durch Reihenentwicklung der Exponentialoperatoren ausgewertet. Für zwei gleichphasig modulierte Impulse $\left(\varphi_{1}=\varphi_{2}=0^{\circ}\right)$ der Längen $\alpha_{1}=90^{\circ}$ und $\alpha_{2}$ ergibt sich beispielsweise bis zu Gliedern 2. Ordnung in $\tau_{1}, \tau_{2}\left(\gg \tau_{a 1}\right.$, $\left.\tau_{\alpha 2}\right)$

$$
\begin{aligned}
U\left(0_{0^{\circ}}^{90^{\circ} \alpha_{2}}\right)= & -\frac{C \sin \omega_{0} t}{\operatorname{Sp} I_{x}{ }^{2}} \operatorname{Sp} I_{y}\left(J_{x} I_{y} J_{x}^{-1}-\frac{\tau_{2}{ }^{2}}{2 ! \hbar^{2}}\left[H^{I I},\left[H^{I I}, J_{x} I_{y} J_{x}{ }^{-1}\right]\right]\right. \\
& \left.-\frac{\tau_{1} \tau_{2}}{\hbar^{2}}\left[H^{I I}, J_{x}\left[H^{I I}, I_{y}\right] J_{x}{ }^{-1}\right]--\frac{\tau_{1}{ }^{2}}{2 ! \hbar^{2}} J_{x}\left[H^{I I},\left[H^{I I}, I_{y}\right]\right] J_{x}{ }^{-1} \cdots\right),
\end{aligned}
$$

$J_{x}=J\left(\begin{array}{c}\alpha_{z} \\ 0^{\circ}\end{array}\right)$. Alle in den $\tau$ ungeraden Glieder verschwinden bei der Spurbildung und sind bereits weggelassen.

Manchmal kann Gl. (1) einfacher als in einer Form nach Gl. (2) ausgewertet werden - insbesondere für Folgen mit mehreren Impulsen -, wenn durch Einschieben von $J J^{-1}=1$ in die Kommutatoren der Entwicklungen geschrieben wird

$$
J\left(\begin{array}{l}
\alpha \\
\varphi
\end{array}\right)\left[H^{I I}, I_{y}\right] J^{-1}\left(\begin{array}{c}
\alpha \\
\varphi
\end{array}\right) \rightarrow\left[J\left(\begin{array}{l}
\alpha \\
\varphi
\end{array}\right) H^{I I} J^{-1}\left(\begin{array}{c}
\alpha \\
\varphi
\end{array}\right), J\left(\begin{array}{l}
\alpha \\
\varphi
\end{array}\right) I_{y} J^{-1}\left(\begin{array}{c}
\alpha \\
\varphi
\end{array}\right)\right] \equiv\left[\widehat{H^{I I}\left(\begin{array}{l}
\alpha \\
\varphi
\end{array}\right)}, \widehat{I_{y}\left(\begin{array}{l}
\alpha \\
\varphi
\end{array}\right)}\right]
$$

und entsprechend bei anderen Ausdrücken. Beispielsweise gelang Alloul und Froidevaux ${ }^{5}$ dadurch die Beschreibung von Modulationen der Einhüllenden von Echos in festen Metallen nach $90^{\circ}-180^{\circ}$.Folgen.

Im weiteren soll jedoch an Beispielen gezeigt werden, daß Umschreibungen nach Gl. (3) nicht immer zu richtigen Ergebnissen führen, weil die Anwendung der Operatoren $H^{I I}$ und $J$ während der Zeiten $\tau$ und $\tau_{\alpha}$ endliche Drehungen der Operatoren $I_{x}, I_{y}$, $I_{z}$ der Kernspins verursachen, deren Reihenfolge im allgemeinen nicht vertauscht werden darf. Bei den hier durchgeführten Rechnungen wurden aber bis zu Gliedern 6. Ordnung in den Zeiten $\tau$ keine Abweichungen von dem Ergebnis gefunden, das ohne die genannte Umformung gewonnen wird, wenn die

\footnotetext{
5 H. Alloul u. C. Froidevaux, Phys. Rev. 163, 324 [1967].
}

Kernspins der Probe den Drehimpuls $I=1 / 2$ und die Impulse bei Phasenverschiebung $n \pi / 2$ die Längen $m \pi / 2(n, m=0,1,2, \ldots)$ haben. Vermutlich bleibt dies auch bei Gliedern noch höherer Ordnung richtig.

Zur Vereinfachung der Darstellung sollen als Beispiele in diesem Abschnitt Ergebnisse für ein Zweispinsystem (nur Paare von Kernspins wechselwirken miteinander durch die dipolare Wechselwirkung) angeführt werden. In diesem Fall ist $[A, B]=0^{2}, H^{I I}$ in Gl. (1) reduziert sich damit auf $H^{I I^{\prime}}=B$.

\section{a) Die Beschränkung auf $I=1 / 2$}

Abweichungen vom exakten Ergebnis durch die Umschreibungen nach Gl. (3) findet man bei Gliedern höherer als 2. Ordnung in den Zeiten $\tau$.

So ergibt sich an Stelle 


$$
\begin{aligned}
& \operatorname{Sp} J\left({ }_{90^{\circ}}^{90^{\circ}}\right)\left[H^{I I^{\prime}},\left[H^{I I^{\prime}}, I_{y}\right]\right] J^{-1}\left({\stackrel{90}{90^{\circ}}}^{\circ}\right)\left[H^{I I^{\prime}},\left[H^{I I^{\prime}}, I_{y}\right]\right]=\operatorname{Sp} \sum_{i<j} b_{i j}{ }^{4}\left(I_{z i}{ }^{4} I_{y j}{ }^{2}+I_{z j}{ }^{4} I_{y i}{ }^{2}\right), \\
& \left.\operatorname{Sp}\left[\widehat{H^{I I^{\prime}}\left(90^{\circ}\right.}\right),\left[\widehat{H^{I I^{\prime}}\left(9_{90^{\circ}}^{\circ}\right)}, \widehat{I_{y}\left(9_{90^{\circ}}^{\circ}\right)}\right]\right]\left[H^{I I^{\prime}},\left[H^{I I^{\prime}}, I_{y}\right]\right]=\mathrm{Sp} \sum_{i<j} b_{i j}{ }^{4}\left(I_{x i}{ }^{2} I_{z i}{ }^{2} I_{y j}{ }^{2}+I_{x j}{ }^{2} I_{z j}{ }^{2} I_{y i}{ }^{2}\right) \text {. }
\end{aligned}
$$

Der durch die Umschreibung entstandene zweite Ausdruck stimmt mit dem ersten nur dann überein, wenn gilt $\mathrm{Sp} I_{\mathrm{Xi}}^{2} I_{\mathrm{Zi}}^{2}=\mathrm{Sp} I_{\mathrm{Zi}}^{4}$, was wegen

Sp $I_{x i}{ }^{2} I_{z i}{ }^{2}=\frac{1}{5}\left(\frac{1}{3} I^{2}(I+1)^{2}+\frac{1}{6} I(I+1)\right)(2 I+1)^{N}$ Sp $I_{z i}{ }^{4}=\frac{1}{5}\left(I^{2}(I+1)^{2}-\frac{1}{3} I(I+1)\right)(2 I+1)^{N}$,

$$
(N=\text { Zahl der Spins } I)
$$

allein für Spins $I=1 / 2 \mathrm{zu}$ erfüllen ist; ebenso gilt nur dann für alle bei Reihengliedern höherer Ordnung auftretenden Ausdrücke

$$
\begin{array}{r}
\mathrm{Sp} I_{a i}^{2 n} I_{\beta i}^{2 m}=\operatorname{Sp} I_{a i}^{2(n+m)}(\alpha, \beta=x, y, \mathrm{z} \\
n, m=0,1,2, \ldots) .
\end{array}
$$

Die in der Literatur entsprechend Gl. (4) abgeleiteten Formeln für Reihenglieder 4. Ordnung gelten demnach trotz Angabe für allgemeine Werte $I$ nur für $I=1 / 2$.

\section{b) Die Beschränkung der Impulslängen}

Die Anwendung der Umschreibung nach Gl. (3) ergibt bei Werten $\alpha \neq m \pi / 2$ für die Drehwinkel der 2 . und folgenden Impulse von Gliedern 6. Ordnung ab Abweichungen vom exakten Ergebnis:

Die Auswertung der Kommutatoren bei der Entwicklung von Gl. (1) liefert stets Ausdrücke der Form $I_{x i}^{a} I_{z i}^{b}(a, b=0,1,2, \ldots)$. Die Wirkung von Impulsoperatoren $J\left(\begin{array}{l}\alpha \\ 0^{\circ}\end{array}\right)=e^{i a I_{x}}, J\left(\begin{array}{l}\alpha \\ 90^{\circ}\end{array}\right)=e^{i a I_{y}}$ hierauf ist wegen $J I_{x i}^{n} I_{x j}^{m} J^{-1}=J I_{x i}^{n} J^{-1} J I_{x j}^{m} J^{-1}$ mit den folgenden Formeln zu bestimmen:

$e^{i \alpha I_{x}} I_{x}^{n} e^{-i \alpha I_{x}}=J\left(\begin{array}{l}\alpha \\ 0\end{array}\right) I_{x}^{n} J^{-1}\left(\begin{array}{l}\alpha \\ 0\end{array}\right)=I_{x}^{n}$,

$J\left(\begin{array}{l}\alpha \\ 0\end{array}\right) I_{y}{ }^{2 n} J^{-1}\left(\begin{array}{c}\alpha \\ 0\end{array}\right)=I_{y}{ }^{2 n}$,

$J\left(\begin{array}{l}\alpha \\ 0\end{array}\right) I_{y}{ }^{2 n+1} J^{-1}\left(\begin{array}{l}\alpha \\ 0\end{array}\right)=I_{y}{ }^{2 n+1} \cos \alpha-I_{z} I_{y}{ }^{2 n} \sin \alpha$,

$J\left(\begin{array}{l}\alpha \\ 0\end{array}\right) I_{z}^{2 n} J^{-1}\left(\begin{array}{l}\alpha \\ 0\end{array}\right)=I_{z}{ }^{2 n}$,

$J\left(\begin{array}{l}\alpha \\ 0\end{array}\right) I_{z}^{2 n+1} J^{-1}\left(\begin{array}{c}\alpha \\ 0\end{array}\right)=I_{z}^{2 n+1} \cos \alpha+I_{y} I_{z}^{2 n} \sin \alpha$

( $n=0,1,2, \ldots$, entsprechende Gleichungen nach zyklischer Vertauschung der Indizes). Gl. (5) gilt nur für Spins $I=1 / 2$ und ist mit Hilfe der Identität $[A, B C]=[A, B] C+B[A, C]$ abzuleiten.

Mit Gl. (5) wird beispielsweise für ein Zweispinsystem

$$
\begin{array}{r}
\mathrm{Sp}\left[H^{I I^{\prime}},\left[H^{I I^{\prime}},\left[H^{I I^{\prime}}, I_{y}\right]\right]\right] J\left(\begin{array}{l}
\alpha \\
0
\end{array}\right)\left[H^{I I^{\prime}},\left[H^{I I^{\prime}},\left[H^{I I^{\prime}}, I_{y}\right]\right]\right] \\
J^{-1}\left(\begin{array}{l}
\alpha \\
0
\end{array}\right)=-X \cos \alpha
\end{array}
$$

für den umgeschriebenen Ausdruck jedoch

$$
\begin{aligned}
& \mathrm{Sp}\left[H^{I I^{\prime}},\left[H^{I I^{\prime}},\left[H^{I I^{\prime}}, I_{y}\right]\right]\right]
\end{aligned}
$$

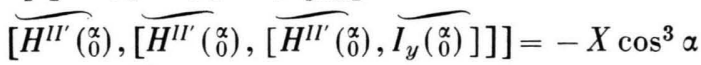

$$
\begin{aligned}
& \left(X=\mathrm{Sp} \sum_{i<j} b_{i j}{ }^{6}\left(I_{x i}{ }^{2} I_{z j}{ }^{6}+I_{x j}{ }^{2} I_{z i}{ }^{6}\right)\right. \text {. }
\end{aligned}
$$

Unterschiede in dieser Form werden erwartet, weil sich nach Gl. (5) lineare Abhängigkeiten der Kommutatoren von $\cos \alpha, \sin \alpha$ ergeben können, während die Form des umgeschriebenen Wechselwirkungsoperators

$$
\begin{aligned}
J\left(\begin{array}{l}
\alpha \\
0
\end{array}\right) & H^{I I^{\prime}} J^{-1}\left(\begin{array}{c}
\alpha \\
0
\end{array}\right)=\widehat{H^{I I^{\prime}}\left(\begin{array}{l}
\alpha \\
0
\end{array}\right)} \\
& =\sum_{i<j} b_{i j}\left(I_{z i} \cos \alpha+I_{y i} \sin \alpha\right)\left(I_{z j} \cos \alpha+I_{y j} \sin \alpha\right)
\end{aligned}
$$

Ergebnisse liefern sollte, die mindestens proportional zu $\cos ^{2} \alpha, \sin ^{2} \alpha, \cos \alpha \sin \alpha$ sind. Bis zu Gliedern 4. und niedrigerer Ordnung in den Zeiten $\tau$ entfallen diese starken Abhängigkeiten aber noch „zufällig“ durch die Möglichkeit, bei Kernspinsystemen mit $I=1 / 2 \cos ^{2} \alpha$ - und $\sin ^{2} \alpha$-Terme zusammenzufassen.

\section{Die Form des Festkörperechos nach zwei Impulsen $\left(\alpha_{1}=\alpha_{2}=90^{\circ}\right)$}

Für das Signal nach 2 Impulsen, die die Kernmagnetisierung um $\alpha_{1}=\alpha_{2}=90^{\circ}$ drehen, deren Dauern $\tau_{a 1}, \tau_{a 2}$ aber vernachlässigbar kurz sind, gilt bei genauer Einhaltung der Resonanzbedingung $\omega_{0}=-\gamma_{I} H_{0}{ }^{1}$ und Phasenverschiebung der Impulsmodulationen $\varphi_{2}-\varphi_{1}=90^{\circ}$

$$
U\left(\begin{array}{c}
90^{\circ} 99^{\circ} \\
0^{\circ}
\end{array}\right) \sim 1-\frac{\left(\tau_{2}^{\prime}-\tau_{1}{ }^{\prime}\right)^{2}}{2 !} M_{2}+\frac{\left(\tau_{2}^{\prime}-\tau_{1}{ }^{\prime}\right)^{4}}{4 !} M_{4}+K
$$

(Definitionen der Momente $M$ vgl. ${ }^{4}, K=$ Korrekturfaktoren, der erste tritt bei der 4. Ordnung auf

$$
K_{4}=\frac{6}{4 !} \tau_{2}{ }^{\prime 2} \tau_{1}{ }^{\prime 2} M_{4}{ }^{\prime}\left(\operatorname{vgl}^{6}{ }^{6}\right) .
$$

Das durch Gl. (6) beschriebene Echo sollte bei Vernachlässigung der Glieder $K$ (exakt gilt dies nur für reine Zweispinsysteme ${ }^{2,7}$ ) seine Maximalamplitude zur Zeit $\tau_{1}{ }^{\prime}=\tau_{2}{ }^{\prime}$ haben. Experimentell findet man jedoch stets beträchtliche systematische Abweichungen. Ein Meßbeispiel zeigt Abb. 2.

\footnotetext{
6 J. G. Powles u. P. Mansfield, Phys. Letters 2, 58 [1962].

7 P. Mansfield, Phys. Rev. 137, A 961 [1965].
} 


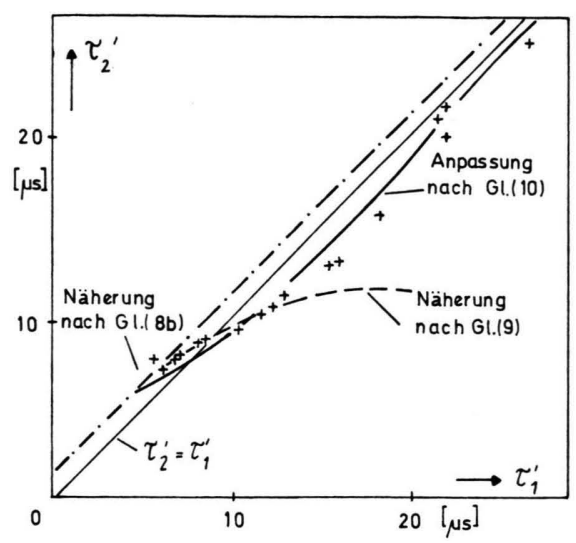

Abb. 2. Lage des Maximums des Echos $U\left({ }^{90^{\circ}} 0^{\circ} 90^{\circ}\right)$ in Abhängigkeit vom Impulsabstand. $+=$ Meßpunkte, $\tau_{\alpha 1}=\tau_{\alpha 2}=2,7 \mu$ s. $\mathrm{C}_{11} \mathrm{H}_{28}$ bei $-100^{\circ} \mathrm{C}$.
Als Ursachen wurden die endlichen Impulslängen und die Glieder $K$ in Gl. (6) in Erwägung gezogen:

\section{a) Wirkung der endlichen Impulsdauern}

Der Einfluß von $H^{I I}$ während der Impulsdauern $\tau_{a 1}, \tau_{a 2} \mathrm{mu}$ jetzt mitberücksichtigt werden, an Stelle

$$
J\left(\begin{array}{l}
\alpha \\
\varphi
\end{array}\right)=\exp \left\{i \alpha\left(I_{x} \cos \varphi+I_{y} \sin \varphi\right)\right\}
$$

ist daher zu setzen

$$
J^{\prime}\left(\begin{array}{c}
\alpha \\
\varphi
\end{array}\right)=\exp \left\{i \alpha\left(I_{x} \cos \varphi+I_{y} \sin \varphi\right)-(i / \hbar) H^{I I}\right\} \text {. }
$$

Da $H^{I I}$ nicht mit $I_{x}$ und $I_{y}$ vertauscht, ist nun auch während der Impulse keine geschlossene Auswertung mehr möglich. Die Rechnung bis zu Gliedern 8. Ordnung in $\alpha_{1}, \alpha_{2}$, aber nur bis zur 2. Ordnung in den Zeiten $\tau_{1}, \tau_{2}$ zwischen und nach den Impulsen ergibt

$$
\begin{aligned}
& U\left(\begin{array}{c}
\alpha_{1} \alpha_{2} \\
0
\end{array} 0^{\circ}\right)=-C \sin \omega_{0} t \sin \alpha_{1}\left\{1-\frac{M_{2}}{2 !}\left(\tau_{2}^{2}+\frac{\tau_{\alpha 2} \tau_{2} \sin 2 \alpha_{2}}{\alpha_{2}}+\frac{\tau_{a 2^{2}} \sin ^{2} \alpha_{2}}{a_{2}{ }^{2}}\right.\right. \\
& \left.+\left(\tau_{\alpha 1}+2 \tau_{1}\right)\left(\tau_{2} \cos 2 \alpha_{2}+\frac{\tau_{\alpha 2}}{2 \alpha_{2}} \sin ^{2} \alpha_{2}\right)+\tau_{\alpha 1} \tau_{1}+\tau_{1}{ }^{2}+L\right\} ; \quad L=\frac{2 \tau_{\alpha 1}{ }^{2}}{\sin \alpha_{1}}\left(\frac{a_{1}}{3 !}-\frac{3 a_{1}{ }^{3}}{5 !}+\frac{6 a_{1}{ }^{5}}{7 !} \ldots\right) ;
\end{aligned}
$$

die zu $\cos \omega_{0} t$ proportionalen Glieder wurden weggelassen, da sie ein hier nicht interessierendes Signal direkt nach dem 2. Impuls beschreiben *

Aus Gl. (7) wird für $\alpha_{1}=\alpha_{2}=90^{\circ}, \tau_{a 1}=\tau_{\alpha 2}=\tau_{\alpha}$

$$
U\left(\begin{array}{c}
90^{\circ} 90^{\circ} \\
0^{\circ} 90^{\circ}
\end{array}\right)=-C \sin \omega_{0} t\left\{1-\frac{M_{2}}{2 !}\left[\left(\tau_{2}-\frac{\tau_{\alpha 1}}{2}-\tau_{1}\right)^{2}+0,5 \tau_{\alpha}^{2}\right]\right\}
$$

und aus $\partial U / \partial \tau_{2}=0$ die Lage des Echomaximums zu

$$
\tau_{2}=\tau_{1}+\frac{\tau_{\alpha 1}}{2} \quad \text { bzw. } \quad \tau_{2}{ }^{\prime}=\tau_{1}{ }^{\prime}-\frac{\tau_{\alpha 1}}{2}+\tau_{\alpha 2}>\tau_{1}{ }^{\prime}
$$

bestimmt.

Die Zeit zwischen den 2 Impulsen wird also scheinbar um $\tau_{a 1} / 2$ verlängert, während die Dauer des 2 . Impulses erwartungsgemäß keinen Einfluß auf $\tau_{2}$ hat, da das Experiment während $\tau_{2}$ die Bedingung des "spin-locking" 9 erfüllt, so daß $H^{I I}$ scheinbar abgeschaltet ist.
Gl. (8 b) deutet die Lage des Echomaximums in Abb. 2 nur für sehr kleine Zeiten.

\section{b) Einfluß der Glieder K}

Der erste Korrekturfaktor wurde von Powles und Strange $^{2}$ zu $M_{4}{ }^{\prime} \approx-0,5 M_{2}{ }^{2}$ abgeschätzt. Nimmt man Gl. $(8 \mathrm{~b})$ auch für Reihenentwicklungen höherer als 2. Ordnung als richtig an, ergibt sich für die Lage des Echomaximums näherungsweise

$$
\tau_{2}^{\prime}=\tau_{1}^{\prime}-\frac{\tau_{\alpha 1}}{2}+\tau_{\alpha 2}-\left(\tau_{1}^{\prime}-\frac{\tau_{\alpha 1}}{2}\right)\left(1-\frac{2 M_{2}}{M_{4}^{\prime}\left(\tau_{1}^{\prime}-\tau_{\alpha 1} / 2\right)^{2}}\right)^{-1}
$$

\footnotetext{
* Mit $\tau_{2}=\tau_{\alpha 2}=0, a_{2}=0^{\circ}, a_{1}=90^{\circ}$ ergibt sich aus Gl. (7) das Signal nach einem Einzelimpuls

$U\left(\begin{array}{c}90^{\circ} \\ 0^{\circ}\end{array}\right)=-C \sin \omega_{0} t\left\{1-M_{2} / 2 !\left[\left(\tau_{1}+\tau_{\alpha 1} / 2\right)^{2}+0,1 \tau_{\alpha 1^{2}}\right]\right\}$

Aus $\partial U / \partial \tau_{1}=0$ findet man den Zeitpunkt $\tau_{1}=-\tau_{\alpha 1} / 2$ für das Signalmaximum, der gleichzeitig als Beginn des Signalabfalls genommen werden muß, damit die Abfälle während und nach dem Impuls stetig ineinander übergehen. - Ein entsprechendes Ergebnis fand bereits BarNaAL ${ }^{8}$, doch fehlt das Korrekturglied $0,1 \tau_{\alpha 1}{ }^{2}$; wahrscheinlich weil die Wir-
}

kungen der von ihm durchgeführten Transformationen, durch die eine Koordinatenachse stets parallel zum momentan herrschenden Magnetfeld ist, nur näherungsweise multiplikativ aneinanderzusetzen sind.

8 D. E. Barnaal, Ph. D. Thesis, University of Minnesota 1965. - D. E. Barnaal u. I. Lowe, Phys. Rev. Letters 11, 258 [1963].

9 S. R. Hartmann u. E. L. Hahn, Bull. Am. Phys. Soc. 5, 498 [1960]. 
Für das Meßbeispiel in Abb. 2 ist $M_{2} \approx 1,25 \cdot 10^{10}$ $\mathrm{s}^{-2}$. Gl. (9) gibt damit den Übergang von $\tau_{2}{ }^{\prime}>\tau_{1}{ }^{\prime}$ zu $\tau_{2}{ }^{\prime}<\tau_{1}{ }^{\prime}$ richtig wieder, doch ist zur Deutung des gemessenen Verlaufs $\tau_{2}{ }^{\prime}=f\left(\tau_{1}{ }^{\prime}\right)$ die Kenntnis der Korrekturglieder wesentlich höherer Ordnung nötig. Diese kann man sich aus dem meßbaren Abfall der Echoamplitude $G\left(\tau_{2}{ }^{\prime}+\tau_{1}{ }^{\prime}-\tau_{\alpha 2}-\tau_{a 1} / 2\right)$ mit wachsendem $\tau_{1}{ }^{\prime}$ verschaffen, der durch die Wirkung dieser Faktoren verursacht ist. Die Form $F^{\prime}$ des Signals ist dann gegeben als

$$
\begin{aligned}
& F^{\prime}=F\left(\tau_{2}{ }^{\prime}-\tau_{1}{ }^{\prime}-\tau_{a 2}+\tau_{a 1} / 2\right) \\
& \cdot G\left(\tau_{2}{ }^{\prime}+\tau_{1}{ }^{\prime}-\tau_{a 2}-\tau_{a 1} / 2\right) .
\end{aligned}
$$

Für das Meßbeispiel in Abb. 2 wird für die Echoform eine Gauß-Funktion angenommen (Halbwertsbreite aus Abfall nach 1. Impuls bestimmt); die Funktion $G$ wurde über die Echoamplituden an den durch Gl. $(8 \mathrm{~b})$ bestimmten Stellen gemessen. Die befriedigende Übereinstimmung des aus Gl. (10) berechneten Kurvenverlaufs mit den Meßpunkten in Abb. 2 zeigt, daß der angenommene Zusammenhang zwischen Echoeinhüllender und Verschiebung des Echomaximums das Experiment beschreiben kann.

\section{Das Signal nach zwei gleichphasig modulierten Impulsen}

Die Auswertung von Gl. (2) für eine $90^{\circ}$ - $\alpha$-Folge gleichphasig modulierter Impulse ergibt nur ein Signal

$$
F\left(\tau_{1}+\tau_{2}\right) \sim 1-\frac{\left(\tau_{1}+\tau_{2}\right)^{2}}{2 !} M_{2},
$$

das unmittelbar nach dem 2 . Impuls erscheint ${ }^{3}\left(\tau_{a 1}\right.$, $\tau_{a 2}$ werden im folgenden gegenüber $\tau_{1}, \tau_{2}$ vernachlässigt).

Dies ist jedoch nur für kleine Werte $\tau_{1}$ erfüllt, bei $\tau_{1} \approx$ Abfallszeit des Signals nach dem 1. Impuls erscheint ein kleines Echo zur Zeit $\tau_{2} \approx \tau_{1}$, wenn $\alpha_{2} \neq 0$, $90^{\circ}$ ist. Seine Amplitudenabhängigkeiten von $\tau_{1}$ und $\alpha_{2}$ zeigen die Abb. 3 und 4. Die Einstellung der Drehwinkel $\alpha$ erfolgte unter Verwendung der $\cos \alpha_{1}$ $\sin \alpha_{2^{-}}$bzw. $\sin \alpha_{1}$-Abhängigkeit für das Signal unmittelbar nach einem 2 . bzw. 1. Impuls ${ }^{3}$.

Durch phasenempfindliche Gleichrichtung ergab sich, daß die Phase des Echos gegenüber dem Signal unmittelbar nach dem 1. und 2. Impuls um $180^{\circ}$ verschoben ist.

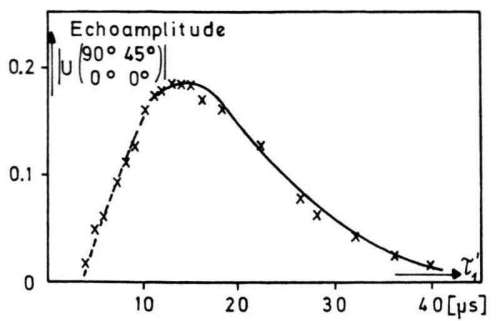

Abb. 3. Abhängigkeit der Amplitude des Echomaximums $U\left(\begin{array}{cc}90^{\circ} & \alpha_{2} \\ 0^{\circ} & 0^{\circ}\end{array}\right)$ vom Impulsabstand bei $\alpha_{2}=45^{\circ}$ (in $\mathrm{C}_{11} \mathrm{H}_{28}$, $\left.-100^{\circ} \mathrm{C}\right)$. Im gestrichelten Bereich liegt das Maximum bei $\tau_{2}{ }^{\prime}>\tau_{1}{ }^{\prime}$. Hier wie in Abb. 4 wurde die maximale Amplitude des Signals $U\left(\begin{array}{c}90^{\circ} \\ 0^{\circ}\end{array}\right)$ gleich 1 gesetzt.

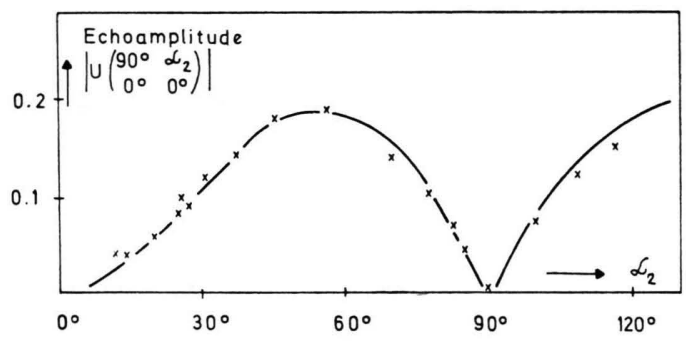

Abb. 4. Abhängigkeit der Amplitude des Echomaximums $U\left(\begin{array}{c}90^{\circ} \alpha_{2} \\ 0^{\circ} 0^{\circ}\end{array}\right)$ vom Drehwinkel $\alpha_{2}$ des 2. Impulses. $x=$ Meßpunkte, ausgezogen: nach Gl. (11) erwarteter Verlauf, Anpassung am Maximum. Impulsabstand $\tau_{1}^{\prime}=13,5 \mu \mathrm{s}=$ const $\left(90^{\circ}\right.$. Impulsdauer $\left.\tau_{\alpha 1}=2,7 \mu \mathrm{s}, \mathrm{C}_{11} \mathrm{H}_{28},-100^{\circ} \mathrm{C}\right)$.

Die zur Deutung durchgeführte Rechnung bis zu Gliedern 6. Ordnung lieferte für $I=1 / 2$

$$
\begin{aligned}
U\left(\begin{array}{c}
90^{\circ} \alpha \\
0^{\circ} 0^{\circ}
\end{array}\right)=-C \sin \omega_{0} t\left\{\operatorname { c o s } \alpha _ { 2 } \left(1-\frac{\left(\tau_{1}+\tau_{2}\right)^{2}}{2 !} M_{2}+\right.\right. & \left.\frac{\left(\tau_{1}+\tau_{2}\right)^{4}}{4 !} M_{4}-\frac{\left(\tau_{1}+\tau_{2}\right)^{2}}{6 !} M_{6}\right) \\
& \left.-\cos \alpha_{2} \sin ^{2} \alpha_{2} \frac{\tau_{1}{ }^{2} \tau_{2}{ }^{2}}{2 ! 2 !}\left(M_{4}{ }^{\prime \prime}+\frac{\left(\tau_{1}-\tau_{2}\right)^{2}}{12} M_{61}{ }^{\prime}+K_{6}\right)\right\}
\end{aligned}
$$

mit

$$
\begin{aligned}
& M_{4}{ }^{\prime \prime}=\frac{1}{N} \sum_{i<j<\alpha}\left(b_{i j} b_{i a}+b_{i j} b_{j a}+b_{i a} b_{j a}\right)^{2}, \\
& M_{61}{ }^{\prime}=-\frac{1}{\cos \alpha_{2} \sin ^{2} \alpha_{2}} \frac{1}{\hbar^{6} \operatorname{Sp} I_{x}{ }^{2}} \operatorname{Sp}\left[H^{I I},\left[H^{I I},\left[H^{I I},\left[H^{I J}, I_{y}\right]\right]\right]\right]\left(J\left(\begin{array}{c}
\alpha \\
0
\end{array}\right)\left[H^{I I},\left[H^{I I}, I_{y}\right]\right] J^{-1}\left(\begin{array}{c}
\alpha \\
0
\end{array}\right)\right.\left.-\cos \alpha_{2}\left[H^{I I},\left[H^{I I}, I_{y}\right]\right]\right), \\
& M_{62}{ }^{\prime}=-\frac{1}{\cos \alpha_{2} \sin ^{2} \alpha_{2}} \frac{1}{\hbar^{6} \mathrm{Sp} I_{x^{2}}{ }^{2}} \operatorname{Sp}\left[H^{I I},\left[H^{I I},\left[H^{I I}, I_{y}\right]\right]\right]\left(J\left(\begin{array}{c}
\alpha \\
0
\end{array}\right)\left[H^{I I},\left[H^{I I},\left[H^{I I}, I_{y}\right]\right]\right] J^{-1}\left(\begin{array}{c}
\alpha \\
0
\end{array}\right)\right.-\cos \alpha_{2}\left[H^{I I},\left[H^{I I},\left[H^{I I}, I_{y}\right]\right]\right], \\
& K_{6}=\tau_{1} \tau_{2}\left(\frac{1}{6} M_{61}{ }^{\prime}+\frac{1}{9} M_{62}{ }^{\prime}\right) .
\end{aligned}
$$


Bei Auswertung der Spur wird $M_{61}^{\prime}$ von $\alpha_{2}$ unabhängig.

Sofern $K_{6}<M_{51}^{\prime}\left(\tau_{1}-\tau_{2}\right)^{2} / 12$ ist, muß nach $\mathrm{Gl}$. (11) an der Stelle $\tau_{1}=\tau_{2}$ ein Echo erscheinen. Die nach Gl. (11) erwartete schnelle Signalvergrößerung mit wachsendem $\tau_{1}$ wurde für kleine $\tau_{1}$ experimentell gefunden (Abb. 3). Das Echomaximum liegt aber nur für Zeiten $\tau_{1} \geqq 11 \mu$ s am erwarteten Zeitpunkt $\tau_{1} \approx \tau_{2}$, unterhalb wirkt sich die additive Überlagerung des $F\left(\tau_{1}+\tau_{2}\right)$-Signalanteils stark aus: Das Maximum des Echos wird dadurch zu Zeiten $\tau_{2}>\tau_{1}$ verschoben, seine Amplitude verringert, und die $\tau_{1}^{2} \tau_{2}^{2}$-Abhängigkeit verfälscht.

Der Einfluß der Länge des 2. Impulses auf die Echoamplitude wurde daher für $\tau_{1}>11 \mu$ s bestimmt. Das Ergebnis (Abb. 4) bestätigt selbst für diesen großen Impulsabstand die berechnete $\cos \alpha_{2} \sin ^{2} \alpha_{2}$ Abhängigkeit.

Bei reinen Zweispinsystemen muß nach Gl. (11) dieses zu $F\left(\tau_{1}+\tau_{2}\right)$ gegenphasige Zusatzsignal völlig verschwinden. Seine Amplitude relativ zu der des Kernsignals unmittelbar nach dem 1 . Impuls ist daher ein Maß für die Größe der Wechselwirkung der Kernspins mit dritten Nachbarn.

\section{Amplituden der Kernsignale bei einer Mehrimpulsfolge}

Besonders eingehend wurde bisher eine Folge untersucht ${ }^{10-12}$, die im Prinzip der sogen. CarrPurcell-Folge ${ }^{13}$ entspricht, die bei Messungen in Flüssigkeiten Anwendung findet: Im Abstand $\tau / 2$ nach einem ersten $90^{\circ}$-Impuls werden weitere $90^{\circ}$. Impulse (Abstand $\tau$ ) eingestrahlt, deren Modulationsphasen gleich und um $\Delta \varphi=90^{\circ}$ bez. der Phase des 1 . Impulses verschoben sind.

Etwa in der Mitte zwischen je 2 Impulsen erscheint dann ein Echo. Die Amplituden der ersten Echos wurden durch Rechnung bis zu Gliedern 4. Ordnung bestimmt ${ }^{14}, W_{A U G H}$ und $W_{A N G}{ }^{12}$ gaben inzwischen Formeln an, die bis zur 6. Ordnung in den Impulsabständen gültig sind.

Für die Amplitude der ersten Echos in der Mitte zwischen den Impulsen, deren Länge vernachlässigt wird, gilt

10 E. D. Ostroff u. J. S. WaUgh, Phys. Rev. Letters 16, 1097 [1966].

11 P. Mansfield u. D. Ware, Phys. Letters 22, 133 [1966]; 23, 421 [1966].

$$
\begin{aligned}
& U\left(\begin{array}{c}
90^{\circ} 90^{\circ} \\
90^{\circ}
\end{array}\right) \sim 1-\frac{\tau^{4}}{64} M_{4}{ }^{\prime}, \\
& U\left({ }^{90^{\circ}} 0^{\circ} 90^{\circ} 90^{\circ} 90^{\circ}\right) \sim 1 \text {, } \\
& U\left(\begin{array}{c}
90^{\circ} 90^{\circ} 90^{\circ} 90^{\circ} 90^{\circ} \\
0^{\circ}
\end{array}\right) \sim 1-\frac{\tau^{4}}{64} M_{4}{ }^{\prime},
\end{aligned}
$$

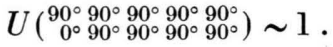

Bemerkenswerterweise entfällt $\mathrm{M}_{4}$ beim 2., 4. und - wie $W_{A U G H}$ und WANG zeigten - auch beim $2 n$ ten Echo, d. h. die Amplituden der 1., 3., 5.... Echos sollten kleiner als die der $2 ., 4 \ldots$ sein, sofern bei ausreichend kleinen Impulsabständen Reihenglieder höherer Ordnung in Gl. (12) noch vernachlässigt werden können.

An einem Gipseinkristall $\left(\mathrm{CaSO}_{4} \cdot 5 \mathrm{H}_{2} \mathrm{O}\right)$, dessen Protonensignal nach einem Einzelimpuls durch geeignete Kristallorientierung im äußeren Magnetfeld sehr langsam abfällt (Halbwertszeit etwa $28 \mu \mathrm{s}$ ), gelang für Impulsabstände $\tau<20 \mu$ s der Nachweis, $\mathrm{da}$ das 2. Echo eine größere Amplitude haben kann als das erste (Abb. 5). Für $\tau>20 \mu$ s ist das zweite Echo jedoch kleiner als das erste; $\tau(\geqq 10 \mu \mathrm{s})$ konnte nicht so klein gemacht werden, daß die folgenden Echos alternierende Amplituden zeigen. Zur Deutung dieser Effekte müssen in Gl. (12) Glieder höherer Ordnung mitgeführt werden.

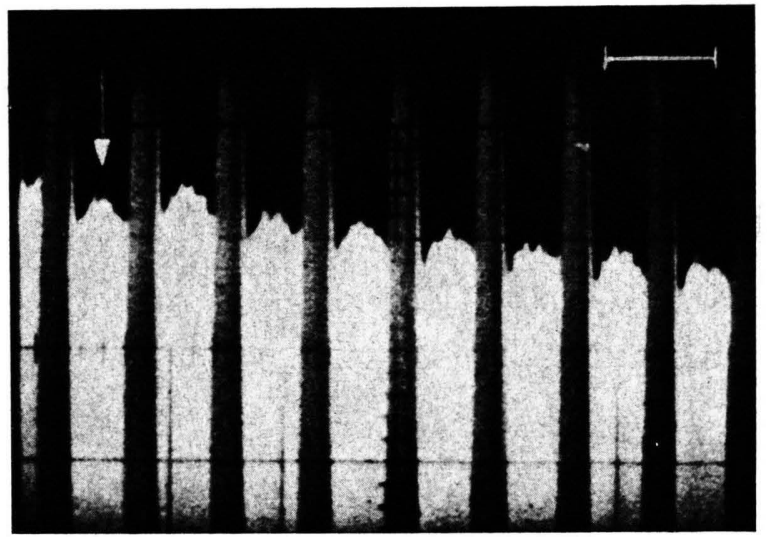

Abb. 5. Echos der im Text angegebenen Vielimpulsfolge. Das erste Echo $(\downarrow)$ hat eine kleinere Amplitude als das zweite

(Gips in Singulettorientierung, $-25^{\circ} \mathrm{C}$ ). $|-| 20 \mu \mathrm{s}$.

\section{Einfluß der Spin-Gitter-Relaxation}

Bei Vergrößerung der Impulsabstände verringern sich die Signalamplituden aller Echos ${ }^{3}$ in vergleich-

12 J. S. W AUgh u. C. H. W $_{\text {ANG }}$, Phys. Rev. 162, 209 [1967].

13 H. Y. Carr u. E. M. Purcell, Phys. Rev. 94, 630 [1954]. D. Gill u. S. Мегвоом, Rev. Sci. Instr. 29, 688 [1958].

14 G. Siegle, Dissertation, Stuttgart 1967. 
baren Zeiten auf Null (typisch $50 \mu \mathrm{s}$ ). Bemerkenswerte Abweichungen hiervon zeigen aber die Signale nach Folgen dieser $90^{\circ}$-Impulse, bei denen die beiden ersten Impulse gleichphasig moduliert sind $\left(\varphi_{1}-\varphi_{2}=0^{\circ}\right)$.

Die Maximalamplitude des erwarteten stimulierten Echos ${ }^{3}$ bei $\varphi_{1}-\varphi_{2}=0^{\circ}, \varphi_{3}-\varphi_{2}=90^{\circ}$ fällt bei Vergrößerung des Abstandes $\tau_{2}$ zwischen dem 2. und 3. Impuls zunächst wie bei wachsendem Abstand $\tau_{1}$ (zwischen 1. und 2. Impuls) schnell $\mathrm{ab}^{15}$, wobei sich die Lage des Echomaximums von $t=\tau_{1}+\tau_{2}$ bis zu $t \approx \tau_{2}$ verschiebt. Aus dem Echo $F\left(\tau_{3}-\tau_{1}\right)$ wird also näherungsweise ein Signal $F\left(\tau_{3}\right)$, Abb. $6 \mathrm{a}, \mathrm{b}$ zeigt ein Beispiel.

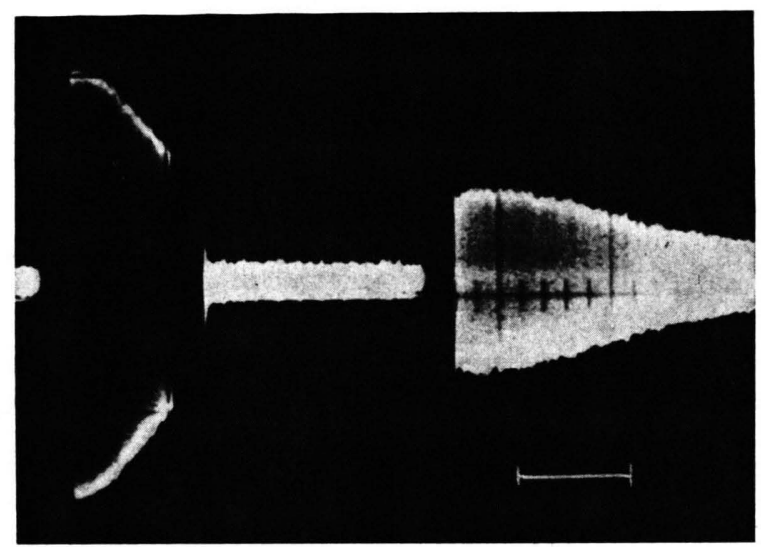

Abb. 6 a.

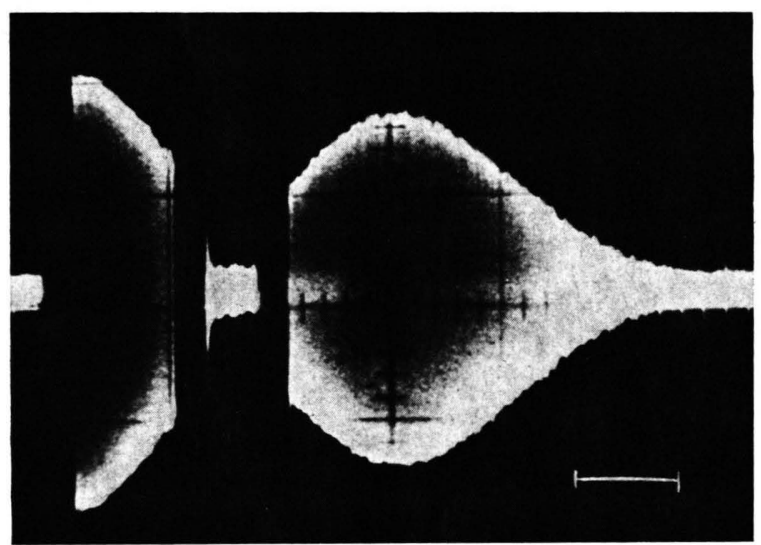

Abb. 6 b.

Abb. 6. Signal $U\left({ }^{90^{\circ}}, 90^{\circ} 90^{\circ}\right)$ von Gips $\left(-20^{\circ} \mathrm{C}\right.$, Singulettorientierung), Impulsabstand in a: $15,5 \mu \mathrm{s}$, in $\mathrm{b}: 45,5 \mu \mathrm{s}$. $\longmapsto \mid 20 \mu \mathrm{s}$.

15 R. Hausser u. G. Siegle, Phys. Letters 19, 356 [1965].
Die Abnahme des Signals $F\left(\tau_{3}\right)$ beim Vergrößern von $\tau_{2}$ kann dann - verglichen mit dem anfänglichen schnellen Abfall des Echos - um mehrere Größenordnungen verlangsamt sein, in guter Näherung gilt

$$
\begin{array}{r}
U\left(\begin{array}{c}
90^{\circ} 90^{\circ} 90^{\circ} \\
0^{\circ} 0^{\circ} 90^{\circ}
\end{array}\right) \sim\left[u e^{-t / T_{1}}+\left(1-e^{-t / T_{1}}\right)\right] F\left(\tau_{3}\right) \\
\left(t \approx \tau_{2} \gg \tau_{1}, \tau_{3}\right),
\end{array}
$$

$u$ ist etwa gleich der Signalamplitude unmittelbar nach Ende des schnellen Signalabfalls und verringert sich rasch bei Vergrößerung des Abstands der beiden ersten Impulse.

Zur Deutung wird für die longitudinale Magnetisierung $M_{z}$ in $z$-Richtung die Gültigkeit der Blochschen Gleichung angenommen

$$
M_{z}(t)=M_{z}\left(t_{0}\right) e^{-t / T_{1}}+\left(1-e^{-t / T_{1}}\right) M_{z 0} .
$$

$M_{z}\left(t_{0}\right)$ bzw. $M_{z 0}$ sind Anfangs- und Gleichgewichtswerte der $z$-Magnetisierung. Gl. (1) bleibt zur Berechnung der Kernsignale weiterhin geeignet, sofern die Signalabfälle in Zeiten erfolgen, die sehr kurz sind verglichen mit $T_{1}$.

Im Anhang wird die Berücksichtigung von Gl. (14) gezeigt, die Auswertung des gewonnenen Ergebnisses [Gl. (A2) ] ergibt

$$
\begin{array}{r}
U\left({ }_{0^{\circ}}^{90^{\circ}} 90^{\circ} 90^{\circ}\right)=-C \cos \omega_{0} t\left[-e^{-t / T_{1}}\left(F\left(\tau_{3}-\tau_{1}\right)+K\right)\right. \\
\left.+\left(1-e^{-t / T_{1}}\right) F\left(\tau_{3}\right)\right] \quad(15)
\end{array}
$$

Die Korrekturfaktoren $K$ verschwinden bemerkenswerterweise auch für ein Zweispinsystem nicht, für den ersten gilt in diesem Fall

$$
K_{4}^{\prime \prime}=-\frac{\tau_{1} \tau_{2}^{2} \tau_{3}}{2 ! 2 ! \hbar^{4} \operatorname{Sp~} I_{x}^{2}} \operatorname{Sp}\left[H^{I I^{\prime}},\left[H^{I I^{\prime}}, I_{y}\right]\right]^{2}<0 .
$$

$K_{4}^{\prime \prime}$ verursacht neben einem Signalabfall auch eine Verschiebung des Echomaximums zu Zeiten $t<\tau_{2}+\tau_{1}$ in Übereinstimmung mit dem Experiment, so daß man bei Berücksichtigung aller Glieder $K$ erwarten kann, daß sich für ausreichend große Zeiten $\tau_{2}$ schließlich $F\left(\tau_{3}-\tau_{1}\right)+K \rightarrow u \mathrm{~F}\left(\tau_{3}\right)$ ergibt.

Herrn Prof. Dr. H. O. Kneser danke ich für die Förderung und Unterstützung der vorliegenden Arbeit; Herrn Dr. F. Noack für viele nützliche Diskussionen. Die Deutsche Forschungsgemeinschaft stellte Sachmittel zur Verfügung. 


\section{Anhang}

\section{Einführung der longitudinalen Relaxationszeit}

Vorausgesetzt wird $\tau_{2} \gg \tau_{1}, \tau_{3} \gg \tau_{\alpha i}(i=1,2,3)$, und der Einfluß der longitudinalen Relaxation daher nur während $\tau_{2}$ berechnet. In Gl. (14) ist die Anfangsmagnetisierung $M_{z 0}=C \operatorname{Sp} I_{z}^{2}$. Zur Zeit $\tau_{1}$ unmittelbar nach zwei gleichphasig modulierten $90^{\circ}$-Impulsen (Dauern werden vernachlässigt) sind die Komponenten der Kernmagnetisierung:

$$
\begin{aligned}
\left\langle M_{x}\left(\tau_{1}\right)\right\rangle & =\left\langle M_{y}\left(\tau_{1}\right)\right\rangle=0, \\
\left\langle M_{z}\left(\tau_{1}\right)\right\rangle & =\frac{C}{\operatorname{Sp} I_{x}{ }^{2}} \operatorname{Sp}\left\{I_{z} J\left({ }_{0^{\circ}}^{90^{\circ}}\right) \exp \left\{-(i / \hbar) H^{I I} \tau_{1}\right\} J\left({ }_{0^{\circ}}^{90^{\circ}}\right) I_{z} J^{-1}\left({ }_{0^{\circ}}^{90^{\circ}}\right) \exp \left\{(i / \hbar) H^{I I} \tau_{1}\right\} J^{-1}\left({ }^{90^{\circ}}\right)\right\} \\
& \equiv \frac{C}{\operatorname{Sp} I_{x}{ }^{2}} \operatorname{Sp} I_{z} \tilde{\sigma}\left(\tau_{1}\right) .
\end{aligned}
$$

$\mathrm{Zu}$ Beginn des 3. Impulses wird daraus mit Gl. (14)

$$
\left\langle M_{z}\left(\tau_{2}\right)\right\rangle=\frac{C}{\operatorname{Sp~} I_{x}^{2}} \operatorname{Sp}\left\{I_{z}^{2}\left(1-e^{-\tau_{2} / T_{1}}\right)+I_{z} \tilde{\sigma}\left(\tau_{1}\right) e^{-\tau_{2} / T_{1}}\right\} \equiv \frac{C}{\operatorname{Sp} I_{x}^{2}} \operatorname{Sp} I_{z} \tilde{\sigma}\left(\tau_{2}\right)
$$

und damit für das Kernsignal nach dem 3. Impuls

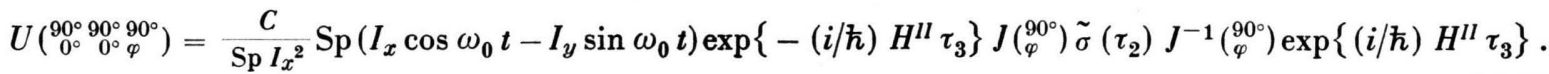

